\title{
Effects of Streptococcus thermophilus on anterior cruciate ligament transection-induced early osteoarthritis in rats
}

\author{
YEN-YOU LIN $^{1 *}$, NAN-FU CHEN ${ }^{2,3 *}$, SAN-NAN YANG $^{4}$, YEN-HSUAN JEAN ${ }^{5}$, HSIAO-MEI KUO ${ }^{6}$,

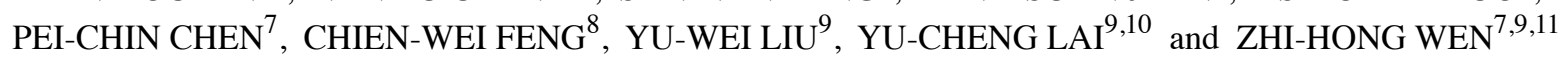 \\ ${ }^{1}$ Department of Sports Medicine, China Medical University, Taichung 40402; ${ }^{2}$ Division of Neurosurgery, \\ Department of Surgery, Kaohsiung Armed Forces General Hospital, Kaohsiung 80284; \\ ${ }^{3}$ Institute of Medical Science and Technology, National Sun Yat-sen University, Kaohsiung 80424; \\ ${ }^{4}$ Department of Pediatrics, E-DA Hospital, School of Medicine, College of Medicine I-Shou University, Kaohsiung 82445; \\ ${ }^{5}$ Department of Orthopedic Surgery, Pingtung Christian Hospital, Pingtung, Pingtung 90059; ${ }^{6}$ Center for Neuroscience, \\ National Sun Yat-sen University, Kaohsiung 80424; ${ }^{7}$ Department of Marine Biotechnology, \\ National Sun Yat-sen University and Academia Sinica, Kaohsiung 80424; ${ }^{8}$ Department of Obstetrics and Gynecology, \\ Kaohsiung Medical University Hospital, Kaohsiung Medical University, Kaohsiung 80756; \\ ${ }^{9}$ Department of Marine Biotechnology and Resources, National Sun Yat-Sen University, Kaohsiung 80424; \\ ${ }^{10}$ Department of Orthopedics, Asia University Hospital, Taichung 41354; ${ }^{11}$ Institute of Biopharmaceutical Sciences, \\ National Sun Yat-sen University, Kaohsiung 80424, Taiwan, R.O.C.
}

Received November 19, 2019; Accepted December 14, 2020

DOI: $10.3892 /$ etm.2021.9653

\begin{abstract}
Osteoarthritis (OA) is the most common joint disorder and is classically defined as a progressively degenerative disease of articular cartilage. It manifests as joint pain and disability and currently has no comprehensive treatments. The primary purpose of the present study was to test the effects of probiotics, Streptococcus thermophilus (TCI633), on anterior cruciate ligament transection (ACLT)-induced experimental osteoarthritis (OA) in rats. In the current study, the experimental groups were given TCI633 $\left(5 \times 10^{9}, 5 \times 10^{10}\right.$ and $5 \times 10^{11} \mathrm{CFU} / \mathrm{kg} /$ day $)$ and glucosamine sulfate $(250 \mathrm{mg} / \mathrm{kg})$ between week 8 and 20 following ACLT. The results showed that oral administration of TCI633 and glucosamine had significant therapeutic effects on pain behaviors and knee swelling. Dose-dependent effects of TCI633 were also observed in ACLT-treated rats. Histopathological analysis demonstrated
\end{abstract}

Correspondence to: Professor Zhi-Hong Wen, Department of Marine Biotechnology and Resources, National Sun Yat-Sen University, 70 Lien-hai Road, Kaohsiung 80424, Taiwan, R.O.C.

E-mail:wzh@mail.nsysu.edu.tw

"Contributed equally

Abbreviations: OA, osteoarthritis; ACLT, anterior cruciate ligament transection; TCI633, Streptococcus thermophilus; OARSI, Osteoarthritis Research Society International

Key words: Streptococcus thermophilus, anterior cruciate ligament transection, glucosamine, Osteoarthritis Research Society International, type-II collagen that ACLT+TCI633 $\left(5 \times 10^{9}, 5 \times 10^{10}\right.$ and $\left.5 \times 10^{11} \mathrm{CFU} / \mathrm{kg} / \mathrm{day}\right)$ improved the synovial inflammation and cartilage damage of ACLT rats. Histology evaluation using the Osteoarthritis Research Society International system and synovial inflammatory score analysis showed the dose-dependent inhibition of TCI633 on synovial inflammation and cartilage damage. Immunohistochemical staining and TUNEL apoptosis staining showed that TCI633 could effectively increase the expression of type II collagen and reduce the amount of chondrocyte apoptosis in cartilage. Therefore, the present study demonstrated that oral intake of TCI633 could significantly suppressing pain behavior, reduce joint swelling and synovial tissue inflammation and increase type II collagen expression in cartilage. There was also a reduction in chondrocyte apoptosis and decreased progression of OA in ACLT-treated rats.

\section{Introduction}

Osteoarthritis (OA) is a degenerative disease that usually occurs in the hands, hips and knees (1). The main causes are mechanical stimulation between bones and aging $(2,3)$.

Cartilage damage is accompanied bone lesions, synovial inflammation and chronic pain, which are the main symptoms of OA (4). In clinical studies, adults $>25$ years old show a prevalence rate of $13.9 \%$ and adults $>65$ years old show a prevalence rate of $33.6 \%$ in the US $(3,5,6)$. For adults $>60$, there is a prevalence rate of $9.6 \%$ in males and $18.0 \%$ in females, worldwide (7). Patients with anterior cruciate ligament (ACL) injury can develop early degeneration of joints (3). Reconstruction surgery can relieve symptoms and reestablish stability of the knee joint, but will not reduce the probability of damage to the ACL progressing to joint degeneration (6). Therefore, OA could be caused by trauma leading to chondrocyte apoptosis 
and chronic pain through biological mechanisms (3). The ACL resection model simulates the development of OA that results from ACL damage (8). This model could be used to assess the health benefits of drugs or supplementary products in relation to degenerative joint disease $(9,10)$. Moreover, this model can be used to study the related factors of OA and drug mechanisms $(11,12)$.

In patients with OA, chronic inflammation primarily occurs in synovial tissue by releasing and increasing the levels of inflammatory factors, such as IL- $1 \beta$ and TNF- $\alpha$, causing the infiltration of immune cells and hyperplasia of the synovial membrane (13). The effects of inflammatory cytokines could cause proteolytic enzymes to degrade type II collagen, proteoglycans and hyaluronic acid on the extracellular matrix of cartilage (14). The extracellular matrix of cartilage is primarily formed through cartilage secretions and plays a critical role in the balance and control of chondrocyte function in cartilage $(15,16)$. Therefore, chondrocyte apoptosis could change the formation of the extracellular matrix of cartilage and become one of the pathological symptoms of OA (16). Chondrocyte apoptosis is mainly induced by signaling pathways that are activated by inflammatory factors, such as IL-1 $\beta$ and TNF- $\alpha(17,18)$. TNF- $\alpha$ induces the TNF-related apoptosis-inducing ligand pathway, in which binding to the death receptors DR4 (TRAIL-RI) and DR5 (TRAIL-RII) induces caspase- $3 / 8$ expression in the intrinsic pathway $(19,20)$. Meanwhile, the extrinsic pathway is mediated by mitochondria with caspase-3/9 regulation (17). However, current treatment of OA mainly focuses on relieving pain symptoms and maintaining or improving joint function (21). Therefore, preliminary treatment of OA uses drugs, such as Panadol or non-steroidal anti-inflammatory drugs (22). Furthermore, moderate $\mathrm{OA}$ is treated with a combination of glucosamine and chondroitin (22). During severe pain or late-stage of the disease, relief of symptoms requires opioids, corticosteroids and hyaluronic acid (22). Finally, consideration is given to surgical methods for artificial joint replacements (22). Annual expenditure for the treatment for OA exceeds \$20 million in the US (23). Hence, it is important to explore treatment methods that can alleviate the disease course of OA.

The present study used the probiotics, Streptococcus thermophilus (TCI633), that can produce hyaluronic acid and be isolated from healthy human breast milk. Previously, TCI633 was identified as a novel strain which was discovered from human breast milk (24). TCI633 can effectively replicate in the gastrointestinal tract (24). Past studies have demonstrated that related bacterial species can suppress allergic reactions, have anti-inflammatory effects and can mediate the immune response $(25,26)$. Moreover, the ability of TCI633 to produce hyaluronic acid is superior to that of known $S$. thermophilus YIT2084 (27). Previously, we demonstrated that TCI633 effectively produces hyaluronic acid in the gastrointestinal tract for absorption and increases hyaluronic acid level in the blood (24). Similarly, a previous study also reported that TCI633 has the potential for the treatment of osteoporosis in vivo (27). Past studies have indicated that hyaluronic acid is a primary glycosaminoglycan in the extracellular matrix where it binds with proteoglycans to lubricate joint cavities and increase the viscosity of synovial fluid $(28,29)$. In the synovial fluid of individuals without OA, the average molecular weight of hyaluronic acid is 4-5 million with a concentration of $2.5-4 \mathrm{mg} / \mathrm{ml}$. Meanwhile, in the synovial fluid of patients with OA, there is an evident decrease in the concentration and molecular weight of hyaluronic acid (28). In previous animal experiments, hyaluronic acid can effectively reduce joint pain and delay disease progression $(30,31)$. Clinical studies have also demonstrated that intra-articular injection of hyaluronic acid can relieve joint pain and improve joint function $(32,33)$. Hyaluronic acid can also regulate the inflammation of $\mathrm{OA}$ through reducing neutrophil chemotaxis, macrophage proliferation and angiogenesis $(34,35)$. Hyaluronic acid can also eradicate proinflammatory cytokines and free radicals from the joint space to lymphatics and reduce cartilage damage (36). Past animal experimentation has also demonstrated that hyaluronic acid can effectively delay the progression of OA in early stage after ACLT (8). Therefore, the purpose of the present study was to investigate the possible therapeutic effects of TCI633 in increasing the level of hyaluronic acid, and to determine if this could alleviate joint pain and swelling and improve joint function in ACLT-treated rats.

\section{Materials and methods}

TCI633, excipient and glucosamine sulfate salt preparation. TCI633 were provided by TCI Co., Ltd. (https://www. tci-bio.com/) and preserved at the Bioresource Collection and Research Center (BCRC), the Food Industry Research and Development Institute (Taiwan) (Species preservation number: BCRC 910636). The excipient and glucosamine sulfate salt (D-glucosamine sulfate salt; cat. no. MG052630801) were also provided by TCI Co., Ltd. The excipient, TCI633 and glucosamine sulfate salt were diluted and suspension in $\mathrm{RO}$ water for oral administration.

Preparation of animals. In total, 63 male Wistar rats (8-weeks old; body weight $320-330 \mathrm{~g}$ ) were obtained from the National Laboratory Animal Centre in Taiwan. The rats were maintained in plexiglass cages in a temperature-controlled $\left(24 \pm 1^{\circ} \mathrm{C}\right)$ room under a 12-h light/dark cycle and given free access to food and water. Each rat was used only once for the experiment. ACLT surgery was performed under $2.5 \%$ isoflurane anesthesia. The use of the animals accorded to the Guiding Principles in the Care and Use of Animals of the American Physiology Society and was approved by the Institutional Animal Care and Use Committee of National Sun Yat-sen University (Kaohsiung, Taiwan; approval no. 10417).

Surgical technique for induction of OA. The method for the rat ACLT model of osteoarthritis was performed according to Ghosh et al (36) and Yang et al (37). A parapatellar incision was made at the right knee and arthrotomy was performed. The ACL was exposed and transected. For the left knee operation, the ACL was exposed, but not transected (37). The pain behaviors and knee swelling were tested once a week. TIC633 was administered from 8th to 20th week for every day after ACLT surgery. TCI633 was no longer administered from weeks 21 to 24 .

Experimental design. Rats were allocated randomly to six groups: Control (n=9), ACLT (n=8), ACLT+TCI633 (5x10 $10^{11} \mathrm{CFU} / \mathrm{kg} /$ day $)$ 
$(\mathrm{n}=8), \mathrm{ACLT}+\mathrm{TCI} 633\left(5 \times 10^{10} \mathrm{CFU} / \mathrm{kg} / \mathrm{day}\right)(\mathrm{n}=9)$, ACLT+TCI633 $\left(5 \times 10^{9} \mathrm{CFU} / \mathrm{kg} /\right.$ day $)(\mathrm{n}=9)$ and ACLT+glucosamine sulfate $(250 \mathrm{mg} / \mathrm{kg})$ group $(\mathrm{n}=9)$. TCI633 and placebo were provided by TCI Co., Ltd. The control group did not receive any treatment. The ACLT group was treated with placebo (oral) with suspension in RO water and ACLT+TCI633 or glucosamine sulfate groups were treated with TCI633 $\left(5 \times 10^{11}, 5 \times 10^{10}\right.$ and $5 \times 10^{9} \mathrm{CFU} / \mathrm{kg} / \mathrm{day}$ suspension in RO water, oral) and glucosamine sulfate $(250 \mathrm{mg} / \mathrm{kg}$ in $\mathrm{RO}$ water, oral) every day from week 8 to week 20 after ACLT surgery. The glucosamine sulfate group was considered as a positive control.

Weight-bearing distribution. The weight bearing distribution for the ACLT-rats was measured using a dual channel weight averager (Singa Technology Corporation), which can independently measure weight bearing of each hind paw $(10,37)$. The change of weight bearing distribution was analyzed as described previously $(9,10,38)$. Briefly, each rat was placed in an angled plexiglass chamber positioned so that each hind paw rested on a separate force plate. The force exerted on each hind paw (measured in grams) was averaged over a 5 -sec period and three measurements were repeated. The changes of hind paw weight distribution are expressed as the difference in weight bearing between the contralateral and ipsilateral (39).

Mechanical allodynia. The method for assessing mechanical allodynia on the rat anterior cruciate ligament transected (ACLT) model of OA was modified from Chaplan et al $(39,40)$. Briefly, rats were placed in compartments of clear plastic cages on top of an elevated metal mesh floor, permitting easy access to paws. A series of von Frey filaments of logarithmically incremental stiffness was applied to the mid-plantar region of the hind paw from below the mesh floor using the 'up-down' method, involving alternate larger and smaller fibers to determine the closest filament to the threshold of pain response (licking or withdrawal).

Measurement of knee width. Knee width was measured to determine the amount of tissue swelling as an index of inflammation. Rats were anesthetized briefly with $2 \%$ isoflurane and then the width of the knee joint was measured every week by using after the ACLT surgery.

Samples collected. Rats were sacrificed by inducing deep anesthesia with $2 \%$ isoflurane. Each rat was then perfused intracardially with ice-cold phosphate-buffered saline (PBS) and $4 \%$ paraformaldehyde at week 24 . The knee samples were collected from rats and fixed in $10 \%$ neutral buffered formalin at room temperature for 3 days, and then decalcified for 2 weeks in buffered 12.5\% EDTA with PBS. The specimens were embedded in paraffin and sliced into $1-\mu \mathrm{m}$ sections. The sections were for hematoxylin/eosin (H\&E) staining at $25^{\circ} \mathrm{C}(2 \mathrm{~min}$ staining with $10 \mathrm{mg} / \mathrm{ml}$ hematoxyli; Mecrk; $15 \mathrm{sec}$ staining with $1 \%$ eosin, Muto Pure Chemicals) and safranin $\mathrm{O} /$ fast green staining at $25^{\circ} \mathrm{C}(10 \mathrm{~min}$ staining with $0.1 \%$ safranin $\mathrm{O}$ and $5 \mathrm{~min}$ staining with $0.1 \%$ fast green, Sigma-Aldrich; Merck KGaA). Each section was examined under an upright light microscope (DM 6000B; Leica Microsystems, Inc.) and digital-image output system (SPOT idea 5.0 Mp Color Digital Camera; Diagnostic Instruments, Inc.).

Histopathological findings of joints. The sections were stained with Safranin-O/fast green and H\&E to assess the general morphology and matrix proteoglycan of the joint tissue. Articular cartilage was graded under microscopic examination according to the Osteoarthritis Research Society International (OARSI) grading system (41). This system comprises of six histological grades and four histological stages. The total score (score $=$ grade $\mathrm{x}$ stage) ranges from 1 point (control articular cartilage) to 24 points (no repair) (41). Synovial tissue was scored with for histological assessment (31). This score assessed i) the synovial tissue, including hyperplasia of the synovial lining cells (0-3 points), hypertrophy of the synovial lining layer (0-3 points) and the infiltration of inflammatory cells (0-3 points), and ii) the sub-synovial tissue, including the proliferation of granulation tissue (0-3 points), vascularization (0-3 points) and infiltration of inflammatory cells (0-3 points). The maximum score is 18 points. The synovitis scores were divided into three stages: 0-6 Points (mild synovitis), 7-12 points (moderate synovitis) and $\geq 13$ or more points (severe synovitis). Higher scores indicated greater damage (31).

Immunohistochemical stain for collagen II. Knee specimens were processed for immunohistochemical analysis, as described in previous studies $(9,10)$. Briefly, the specimens were de-paraffinized with xylene and rehydrated with alcohol, and the endogenous peroxidase activity was quenched by $0.3 \%$ hydrogen peroxide. Antigen retrieval was performed using $20 \mathrm{mM}$ proteinase K (Sigma-Aldrich; Merck KGaA) in PBS for $40 \mathrm{~min}$, and slides were incubated with primary antibody, polyclonal anti-rabbit anti-collagen II (1:200; cat. no. 234187; Calbiochem; Merck KGaA). Then, the sections were incubated with second antibody anti-rabbit IgG (Vector Laboratories, Inc.). Specimens were labeled with the avidin-biotin complex technique using an $\mathrm{ABC}$ kit (Vectastain $\mathrm{ABC}$ kit; Vector Laboratories Inc.). Finally, specimens were treated with 3,30-diaminobenzidine tetrahydrochloride (DAB; peroxidase substrate kit; Vector Laboratories Inc.) for $2 \mathrm{~min}$. Slides were analyzed under a light microscope with a microscope digital image output system. The quantitation of type II collagen immunoreactivity in cartilage was analyzed by ImageJ version 1.51j8 (National Institutes of Health) with x200 magnification images, and the results were averaged.

TUNEL staining. The sections were taken from the knee joint for apoptosis dyeing of chondrocytes. Briefly, the paraffin was removed and sections were rehydrated by soaking in xylene (100\%), alcohol (100, 95, 75 and 50\%), and then proteinase K ( $20 \mathrm{mM}$; Sigma-Aldrich; Merck KGaA) was added for $30 \mathrm{~min}$ and rinsed with PBS. Hydrogen peroxide (3\%) solvent was added for $10 \mathrm{~min}$. Sections were permeabilized with permeabilization solution containing $0.1 \%$ Triton $\mathrm{X}-100$ and $0.1 \%$ sodium citrate for a $30 \mathrm{~min}$ reaction at room temperature and rinsed with PBS. Horse serum (3\%, Jackson ImmunoResearch Inc.) was added for $60 \mathrm{~min}$ and the TUNEL reaction mixture agent (TUNEL reaction mixture; In Situ Cell Death Detection kit, Fluorescein; cat. no. 11684795910, Sigma-Aldrich; 
Merck KGaA) for a $90-$ min reaction at $37^{\circ} \mathrm{C}$. Samples were stained with DAB dye at $25^{\circ} \mathrm{C}$ for $5 \mathrm{~min}$, and then soaked in $10 \mathrm{mg} / \mathrm{ml}$ hematoxylin stai (Merck $\mathrm{KGaA}$ ) at $25^{\circ} \mathrm{C}$ for nuclear staining for $90 \mathrm{sec}$. Slides were analyzed under a light microscope with a microscope digital image output system. The percentage of TUNEL-positive cells were quantified by determining the number of positive chondrocytes with $\mathrm{x} 200$ magnification images, as previously described (42).

Statistical analysis. All data are expressed as mean \pm standard error of the mean in triplicate, unless otherwise specified. For statistical analysis, all data were analyzed the differences between the experimental groups using a standard two-way ANOVA and one-way ANOVA followed Tukey's post hoc test at the same time-point for multiple comparisons (SigmaPlot 11.0 for Windows; Systat Software, Inc.). $\mathrm{P}<0.05$ was considered to indicate a statistically significant difference.

\section{Results}

Effects of TCI633 on mechanical allodynia. Evaluation of the treatment effects of TCI633 in ACLT-induced mechanical allodynia showed that ACLT significantly induced mechanical allodynia with the significant difference of reduction threshold between the ACLT group and Control group between weeks 7 to 24 (Fig. 1A). The changes of mechanical allodynia were analyzed using ANOVA for repeated measures, and the results showed significant effects in the treatment groups and time. There was a statistically significant interaction between treatment and time (data not shown). Comparison of the ACLT+TCI633 (5×10 11 CFU/kg/day) and ACLT group showed a significant increase of paw withdrawal threshold between weeks 10 to 24 after ACLT surgery (Fig. 1A). Comparison of the ACLT+TCI633 (5x10 $\left.{ }^{10} \mathrm{CFU} / \mathrm{kg} / \mathrm{day}\right)$ group and ACLT group showed a significant increase of threshold between weeks 11 and 22 (Fig. 1A). Comparison of the ACLT+TCI633 (5×10 ${ }^{9} \mathrm{CFU} / \mathrm{kg} /$ day) group and ACLT group showed a significant increase of threshold between weeks 15 to 17 and 19 to 20 (Fig. 1A). Comparison of the ACLT+glucosamine and ACLT group showed a significant increase of threshold between weeks 11 to 24 after ACLT surgery (Fig. 1A). Moreover, comparison of the ACLT+TCI633 $\left(5 \times 10^{10}\right.$ or $5 \times 10^{11} \mathrm{CFU} / \mathrm{kg} /$ day) group and ACLT ACLT+TCI633 $\left(5 \times 10^{9} \mathrm{CFU} / \mathrm{kg} /\right.$ day) group showed a significant increase of threshold. Therefore, these results suggested that TCI633 improves the paw withdrawal threshold of ACLT-induced mechanical allodynia with dose-dependent effects. Once TCI633 was no longer administered (weeks 21 to 24), there was a downward trend for pain relief effects on mechanical allodynia in all ACLT+TCI633 groups.

Effects of TCI633 on weight bearing. Evaluation of the treatment effects of TCI633 on ACLT-induced differences of weight bearing showed that ACLT significantly induced differences of weight bearing distribution between the ACLT group and Control group between weeks 1 to 24 (Fig. 1B). The changes of weight bearing were analyzed using ANOVA for repeated measures, and the results showed significant effects in the treatment groups and time. There was a statistically significant interaction between treatment and time.
Comparison of the ACLT+TCI633 (5x10 11 CFU/kg/day) and ACLT group showed the significant decrease of the difference of weight bearing between weeks 9 to 24 after ACLT surgery (Fig. 1B). Comparison of the ACLT+TCI633 $\left(5 \times 10^{10} \mathrm{CFU} / \mathrm{kg} /\right.$ day) and ACLT groups showed a significant decrease in the difference of weight bearing between weeks 10 to 21 and weeks 22 to 24 (Fig. 1B). Comparison of the ACLT+TCI633 (5x10 $\mathrm{CFU} / \mathrm{kg} /$ day) and ACLT groups showed a significant decrease in the difference of weight bearing between weeks 11 to 17 and week 21 (Fig. 1B). Comparison of the ACLT+glucosamine $(250 \mathrm{mg} / \mathrm{kg})$ and ACLT groups showed a significant decrease in the difference of weight bearing between weeks 9 to 24 (Fig. 1B). Moreover, comparison of the ACLT+TCI633 $\left(5 \times 10^{10}\right.$ or $5 \times 10^{11} \mathrm{CFU} / \mathrm{kg} /$ day $)$ and ACLT+TCI633 $\left(5 \times 10^{9} \mathrm{CFU} / \mathrm{kg} /\right.$ day) groups showed a significant decrease in the difference of weight bearing. Therefore, these results demonstrated that TCI633 improved the effects of ACLT on weight bearing in a dose-dependent manner. Once TCI633 was no longer administered (weeks 21 to 24), there was an upward trend for pain relief effects on weight bearing in all ACLT+TCI633 groups.

Effects of STCI633 on knee swelling. Evaluation of the treatment effects of TCI633 in ACLT-induced knee swelling showed that ACLT significantly induced the swelling of the knee compared with the Control group between weeks 1 to 24 (Fig. 1C). Comparison of the ACLT+TCI633 $\left(5 \times 10^{11} \mathrm{CFU} / \mathrm{kg} /\right.$ day $)$ and ACLT groups showed a significant decrease of swelling of the knee between weeks 11 to 23 after ACLT surgery (Fig. 1C). The changes of knee swelling were analyzed by ANOVA for repeated measures, and the results showed significant effects in the treatment groups and time. There was a statistically significant interaction between treatment and time. Comparison of the ACLT+TCI633 (5×10 $\left.{ }^{10} \mathrm{CFU} / \mathrm{kg} / \mathrm{day}\right)$ ACLT group showed a significant decrease of swelling of the knee between weeks 12 and weeks 14 to 21 (Fig. 1C). Comparison of the ACLT+TCI633 (5x10 ${ }^{9} \mathrm{CFU} / \mathrm{kg} /$ day) and ACLT groups showed a significant decrease of swelling of the knee between weeks 14 and weeks 16 to 21 (Fig. 1C). Comparison of the ACLT+glucosamine $(250 \mathrm{mg} / \mathrm{kg})$ and ACLT groups showed a significant decrease of swelling of the knee between weeks 11 to 24 (Fig. 1C). Moreover, comparison of the ACLT+TCI633 $\left(5 \times 10^{10}\right.$ or $5 \times 10^{11} \mathrm{CFU} / \mathrm{kg} /$ day) and ACLT ACLT+TCI633 (5x10 ${ }^{9} \mathrm{CFU} / \mathrm{kg} /$ day) groups showed a significant decrease of swelling of the knee. In summary, these results verified that TCI633 improved the effects on ACLT-induced swelling of the knee with dose-dependent effects. Once TCI633 was no longer administered (weeks 21 to 24), there was an upward trend for pain relief effects on knee swelling from all ACLT+TCI633 groups.

Effects of TCI633 on body weight change. The effect of TCI633 on body weight changes in ACLT models (Fig. 1D) was analyzed using ANOVA for repeated measures. The results showed significant effects in the treatment groups and time. There was also a statistically significant interaction between treatment and time. Results showed that in terms of weight change, the ACLT and Control groups were not significantly different from weeks 0 to 24, although a time-dependent increase in weight was observed. After ACLT surgery, comparison of 

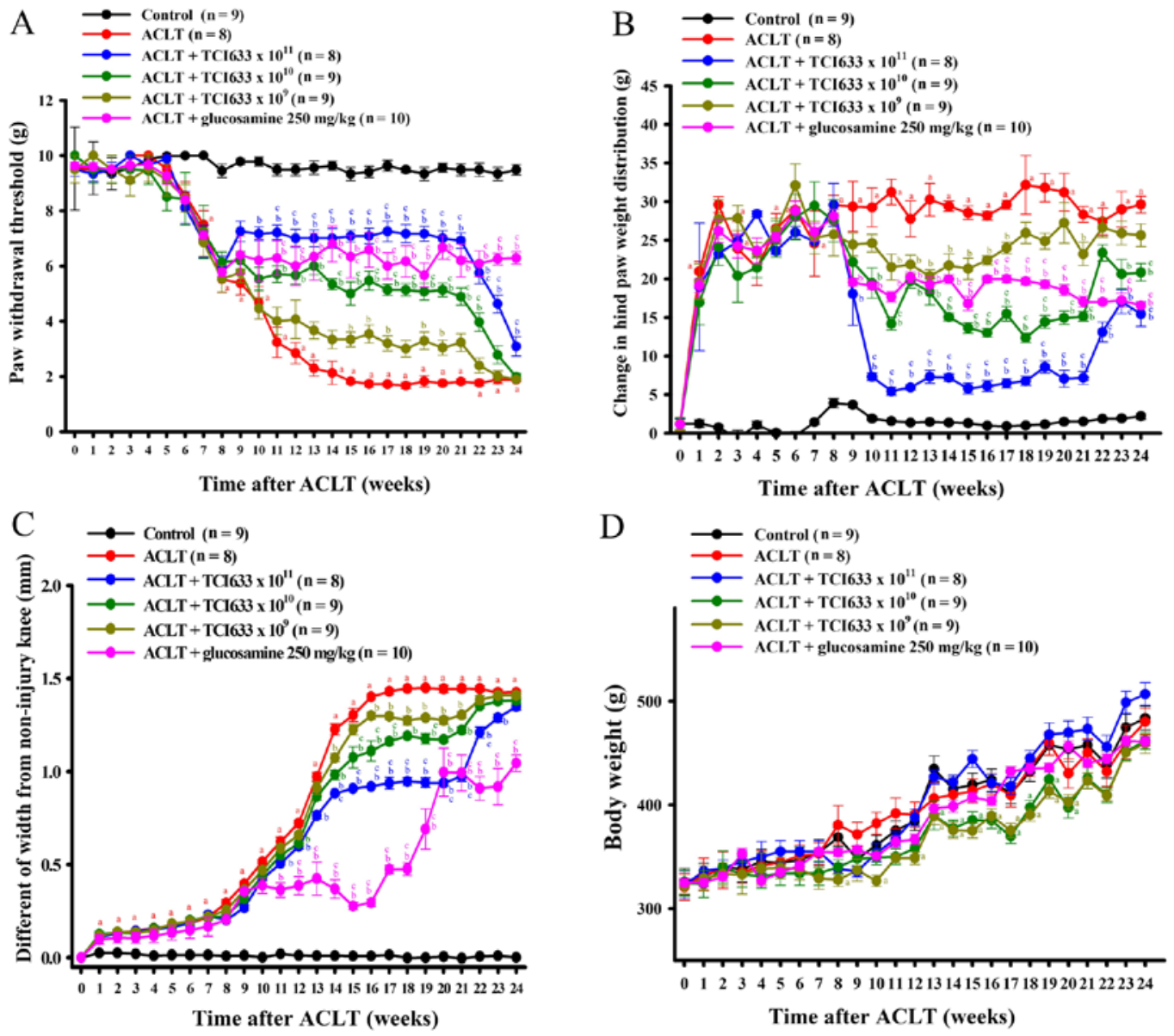

Figure 1.Effects of TCI633 in ACLT induced OA model. Effect of TCI633 on (A) mechanical allodynia,(B) changes of weight bearing, (C) knee joint swelling and (D) body weight. ${ }^{a} \mathrm{P}<0.05$ ACLT vs. Control groups at the same time-point; ${ }^{\mathrm{b}} \mathrm{P}<0.05 \mathrm{ACLT}+$ treatment (including TCI $633 \times 10^{9}, \mathrm{x} 10^{10}$ and $\times 10^{11} \mathrm{CFU} / \mathrm{kg} / \mathrm{day}$ and glucosamine) vs. ACLT groups at the same time-point; ${ }^{\mathrm{P}} \mathrm{P}<0.05 \mathrm{ACLT}+$ treatment (including TCI633 $\times 10^{10}$ and $\times 10^{11} \mathrm{CFU} / \mathrm{kg} /$ day and glucosamine) vs. ACLT+TCI633 $5 \times 10^{9} \mathrm{CFU} / \mathrm{kg} /$ day group at the same time-point. TCI633, Streptococcus thermophilus; ACLT, anterior cruciate ligament transection.

the ACLT+TCI633 (5x10 $11 \mathrm{CFU} / \mathrm{kg} / \mathrm{day})$ and ACLT groups showed no significant changes in weight from weeks 0 to 24 with a time-dependent increase in weight in both groups. Comparison of the ACLT+TCI633 (5x10 10 CFU/kg/day) and Control groups showed a significant decrease in weight from weeks 13 to 20 (Fig. 1D). Comparison of the ACLT+TCI633 (5x10 $\mathrm{CFU} / \mathrm{kg} / \mathrm{day})$ and Control groups showed a significant weight decrease from weeks 8 to 10 and weeks 13 to 20 (Fig. 1D). Comparison of the ACLT+glucosamine and ACLT groups showed no significant weight change from weeks 0 to 24 . In summary, these results verified the effect of TCI633 on weight change in ACLT models, with a slight delay in weight increase in the ACLT+TCI633 $\left(5 \times 10^{10}\right.$ and $5 \times 10^{9} \mathrm{CFU} / \mathrm{kg} /$ day) groups.

Effect of TCI633 on synovial inflammation. Evaluation of the effect of TCI633 on synovial inflammation in ACLT-treated rats compared with Control rats showed infiltration of inflammatory cells, tissue hyperplasia and hypertrophy (black arrows) in synovial tissue in week 24 (Fig. 2A). Comparison of the ACLT+TCI633 (5x10 ${ }^{9} \mathrm{CFU} / \mathrm{kg} /$ day) and ACLT groups demonstrated a similar phenomenon. Comparison of the ACLT+TCI633 $\left(5 \times 10^{11}\right.$ or $5 \times 10^{10} \mathrm{CFU} / \mathrm{kg} /$ day $)$ and ACLT groups revealed slight inflammation with fewer inflammatory cells and improvement of synovial tissue hyperplasia and hypertrophy at week 24 after ACLT (Fig. 2A). When evaluating the effects of TCI633 on synovial inflammation of ACLT rats using histopathological analysis, the ACLT group showed a significant increase in synovial score compared with the Control group (Fig. 2B). The ACLT+TCI633 (5x10 11 and $5 \times 10^{10} \mathrm{CFU} / \mathrm{kg} / \mathrm{day}$ ) and ACLT+glucosamine groups showed a significant decrease in synovial score compared with ACLT group (Fig. 2B). Moreover, there was a significant decrease in synovial score in ACLT+TCI633 $\left(5 \times 10^{11} \mathrm{CFU} / \mathrm{kg} /\right.$ day $)$ and ACLT+glucosamine compared with the ACLT+TCI633 (5x10 $\left.{ }^{9}\right)$ group (Fig. 2B). In summary, these results verified that TCI633 and glucosamine significantly reduced the effects of synovial tissue inflammation after ACLT.

Effects of TCI633 on cartilage damage. Evaluation of the effects of TCI633 on the cartilage of knee after ACLT showed that, compared with the Control group, ACLT resulted in obvious damage to surface of cartilage and reduction of chondrocyte number. Safranin O/fast green staining showed decreased signals (red) reflecting the intensity of the cartilage layer in week 24 (Fig. 3A). While comparing the ACLT+TCI633 or ACLT+glucosamine groups with the ACLT group, there were slight irregularities on the surface of cartilage and a slight decrease in the intensity of staining signal in cartilage was observed (Fig. 3). There was a significant increase in OARSI 
A

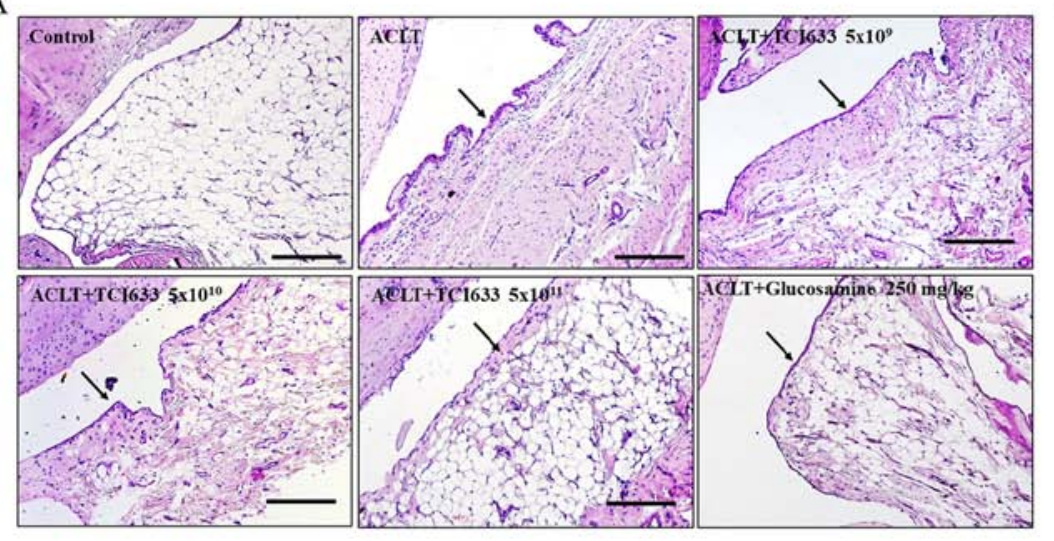

B

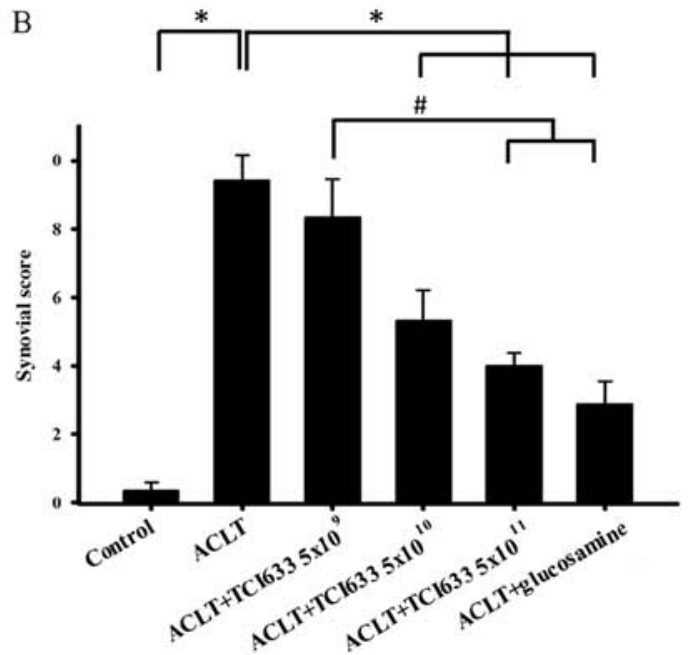

Figure 2. Histological evaluation of TCI633 on synovial tissue inflammation. (A) Representative hematoxylin staining images of the ankle joint sections from Control, ACLT, ACLT+TCI633 $\left(5 \times 10^{9}, 5 \times 10^{10}\right.$ and $5 \times 10^{11} \mathrm{CFU} / \mathrm{kg} /$ day $)$ and ACLT+glucosamine $(250 \mathrm{mg} / \mathrm{kg})$ groups. Magnification, $\mathrm{x} 100$. Scale bar, $200 \mu \mathrm{m}$. Arrow indicated the hypertrophy of synovial tissue. (B) Synovial inflammatory score. "P<0.05 vs. ACLT; ${ }^{\# P}<0.05$ ACLT+TCI633 (5x10 10 or $\left.5 \times 10^{11} \mathrm{CFU} / \mathrm{kg} / \mathrm{day}\right)$ or glucosamine groups vs. ACLT+TCI633 $\left(5 \times 10^{9} \mathrm{CFU} / \mathrm{kg} / \mathrm{day}\right)$ group. TCI633, Streptococcus thermophilus; ACLT, anterior cruciate ligament transection.

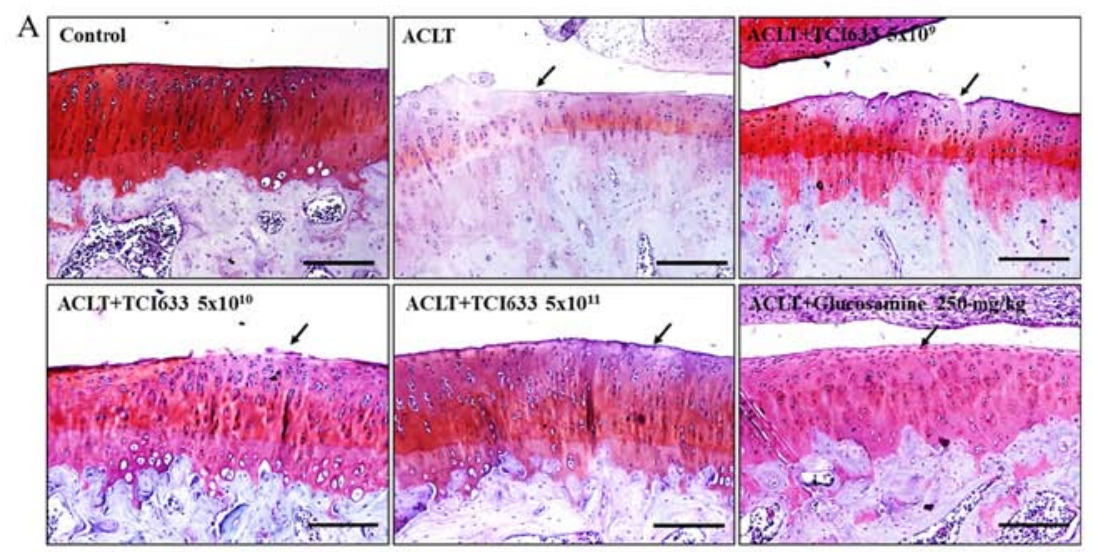

B

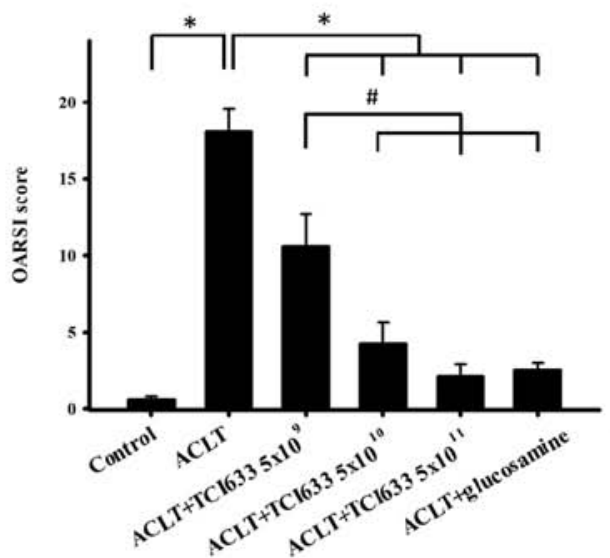

Figure 3. Histological evaluation of TCI633 on cartilage damage. (A) Safranin O and fast green staining images of the ankle joint sections from Control, ACLT, ACLT+TCI633 $\left(5 \times 10^{9}, 5 \times 10^{10}\right.$ and 5x10 ${ }^{11} \mathrm{CFU} / \mathrm{kg} /$ day) and ACLT+glucosamine $(250 \mathrm{mg} / \mathrm{kg})$ groups. Magnification, x100. Scale bar, $200 \mu \mathrm{m}$. (B) OARSI scores in different treatment groups. ${ }^{*} \mathrm{P}<0.05$ vs. ACLT; ${ }^{*} \mathrm{P}<0.05$ ACLT+TCI633 (5x10 10 or $\left.5 \times 10^{11} \mathrm{CFU} / \mathrm{kg} / \mathrm{day}\right)$ or glucosamine groups vs. ACLT+TCI633 (5x10 $\left.\mathrm{CFU} / \mathrm{kg} / \mathrm{day}\right)$ group. TCI633, Streptococcus thermophilus; ACLT, anterior cruciate ligament transection; OARSI, Osteoarthritis Research Society International.

score between the ACLT and Control groups (Fig. 3B). There was a significant decrease in OARSI score in ACLT+TCI633 and glucosamine groups with compared with ACLT group. Moreover, there was a significant decrease in OARSI score in ACLT+TCI633 $\left(5 \times 10^{10}\right.$ or $5 \times 10^{11} \mathrm{CFU} / \mathrm{kg} /$ day $)$ and ACLT+glucosamine groups compared with the ACLT+TCI633 $\left(5 \times 10^{9}\right)$ group. These results verified that TCI633 reduced the damage of cartilage after ACLT.

Effect of TCI633 in type II collagen expression of cartilage. The effects of TCI633 on type II collagen expression in the cartilage of ACLT-treated rats were evaluated. Comparison of the ACLT and Control groups showed a notable reduction in type II collagen expression in the cartilage after ACLT (Fig. 4). The ACLT+TCI633 $\left(5 \times 10^{9} \mathrm{CFU} / \mathrm{kg} /\right.$ day) group also displayed similar conditions compared with the ACLT group (Fig. 4).
In the ACLT+TCI633 $\left(5 \times 10^{10}\right.$ or $5 \times 10^{11} \mathrm{CFU} / \mathrm{kg} /$ day $)$ and ACLT+glucosamine groups, there was an increasing trend in type II collagen expression compared with the ACLT group. Quantitative analysis of type II collagen expression in cartilage demonstrated that the ACLT group had a significant decrease in immunoreactivity compared with the Control group (Fig. 5B). In the ACLT+TCI633 $\left(5 \times 10^{10}\right.$ or $5 \times 10^{11} \mathrm{CFU} / \mathrm{kg} /$ day) and ACLT+glucosamine groups, there was a significant increase in immunoreactivity compared with the ACLT group. Moreover, there was a significant increase in immunoreactivity in the ACLT+TCI633 $\left(5 \times 10^{10}\right.$ or $5 \times 10^{11} \mathrm{CFU} / \mathrm{kg} / \mathrm{day}$ ) and ACLT+glucosamine groups compared with the ACLT+TCI633 $\left(5 \times 10^{9} \mathrm{CFU} / \mathrm{kg} /\right.$ day $)$ group (Fig. 4B). In the ACLT+glucosamine group there was a similar effect compared with the ACLT+TCI633 (5x10 $10^{11} \mathrm{CFU} / \mathrm{kg} /$ day) group (Fig. 4B). These results suggested 

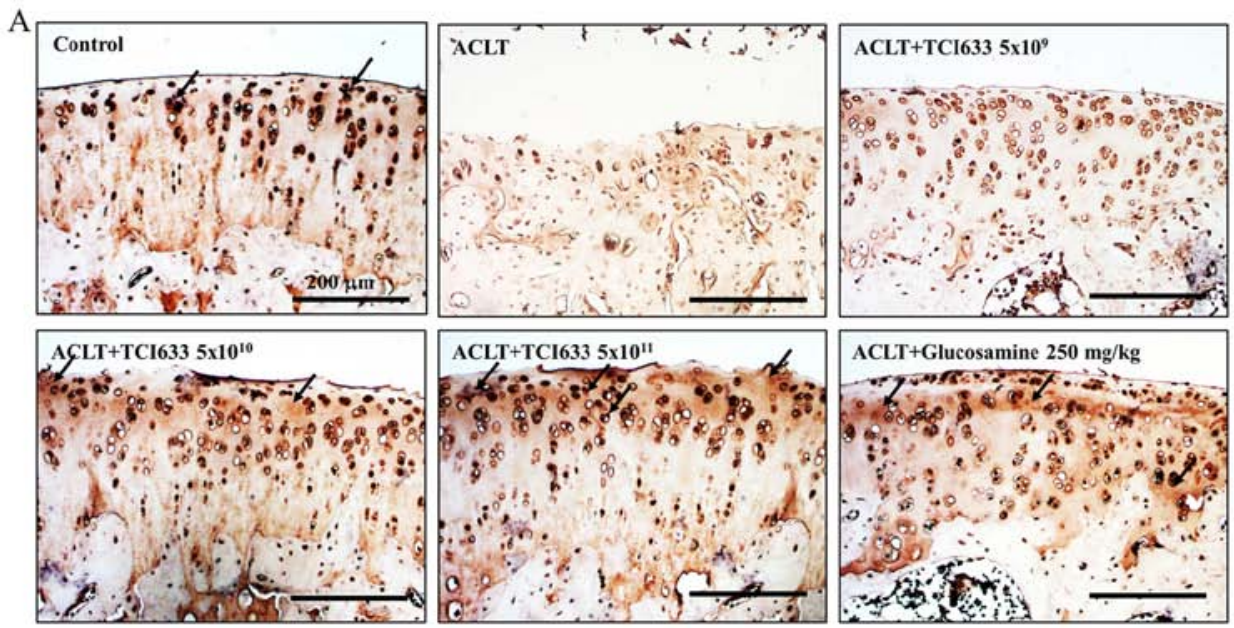

B

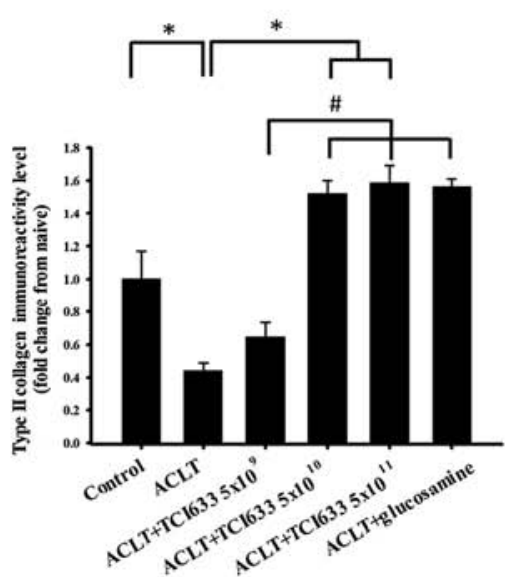

Figure 4. Evaluating the effect of tci633 on type II collagen in cartilage. (A) Immunoreactivity of type II collagen is shown in red-brown (arrow) of cartilage from Control, ACLT, ACLT+TCI633 $\left(5 \times 10^{9}, 5 \times 10^{10}\right.$ or $5 \times 10^{11} \mathrm{CFU} / \mathrm{kg} /$ day $)$ and ACLT+glucosamine $(250 \mathrm{mg} / \mathrm{kg})$ groups. Magnification, $\mathrm{x} 200$. Scale bar, $200 \mu \mathrm{m}$. (B) Quantitative analysis of type II collagen expression in the cartilage. ${ }^{*} \mathrm{P}<0.05$ vs. ACLT; ${ }^{~} \mathrm{P}<0.05$ ACLT+TCI633 (5x $10^{10}$ or $\left.5 \times 10^{11} \mathrm{CFU} / \mathrm{kg} / \mathrm{day}\right)$ or glucosamine groups vs. ACLT+TCI633 (5x10 $\mathrm{CFU} / \mathrm{kg} / \mathrm{day})$ group. TCI633, Streptococcus thermophilus; ACLT, anterior cruciate ligament transection.


Figure 5. Evaluating the effect of TCI633 in chondrocyte apoptosis. (A) TUNEL staining is shown in red-brown (arrow) of cartilage from Control, ACLT, ACLT+TCI633 $\left(5 \times 10^{9}, 5 \times 10^{10}\right.$ or $5 \times 10^{11} \mathrm{CFU} / \mathrm{kg} /$ day $)$ and ACLT+glucosamine $(250 \mathrm{mg} / \mathrm{kg})$ groups. Magnification, x200. Scale bar, $200 \mu \mathrm{m}$. (B) Quantitative analysis of TUNEL staining positive cells of the cartilage. ${ }^{*} \mathrm{P}<0.05$ vs. ACLT; ${ }^{~} \mathrm{P}<0.05$ ACLT+TCI633 $\left(5 \times 10^{10}\right.$ or $5 \times 10^{11} \mathrm{CFU} / \mathrm{kg} / \mathrm{day}$ ) or glucosamine groups vs. ACLT+TCI633 (5x10 $\mathrm{CFU} / \mathrm{kg} /$ day) group. TCI633, Streptococcus thermophilus; ACLT, anterior cruciate ligament transection.

that TCI633 increased type II collagen expression in the cartilage of ACLT-rats.

Effects of TCI633 on chondrocyte apoptosis of cartilage. The effect of TCI633 in protecting chondrocytes in the ACLT model was evaluated (Fig. 5). Under Control conditions, only a small ratio of chondrocytes undergo apoptosis (Fig. 5B) The results showed that there was an evident condition of chondrocyte apoptosis in week 24 after ACLT. With comparing with the ACLT group, there was a reduction of chondrocyte apoptosis observed in TCI633 $\left(5 \times 10^{10}\right.$ or $5 \times 10^{11} \mathrm{CFU} / \mathrm{kg} /$ day $)$ and glucosamine treatment groups (Fig. 5B). Quantitative percentage analysis showed that there was a clear increase in the percentage of TUENL-positive chondrocytes in the ACLT group with compared the Control groups in week 24. When comparing the TIC633 and glucosamine treatment groups with the ACLT group, both show a clear reduction in the percentage of TUNEL-positive cells. When comparing the three different TIC633 dose groups with the ACLT group, there is the dose-dependent effect of the reduction in the percentage of TUNEL-positive cells. Moreover, there was a significant decrease in the percentage of TUNEL-positive cells in the ACLT+TCI633 (5x10 $\left.{ }^{11} \mathrm{CFU} / \mathrm{kg} / \mathrm{day}\right)$ groups and ACLT+glucosamine groups compared with the ACLT+TCI633 (5x $10^{9} \mathrm{CFU} / \mathrm{kg} /$ day) group (Fig. 5B). These results suggested that TCI633 significantly protected chondrocytes against apoptosis in the ACLT model.

\section{Discussion}

The purpose of the present study was to examine the possible effects of TCI633 on OA using an ACLT-induced OA rat 
model. A previous in vivo study have demonstrated that earlier administration of hyaluronic acid could delay the progression of OA in an ACLT rat model (8). Previous studies have reported that TCI633 can effectively produce hyaluronic acid in the gastrointestinal tract for absorption through the intestines, resulting in increased hyaluronic acid content in the blood and showing potential for treating osteoporosis $(24,27)$. The present results showed that TCI633 can effectively alleviate the pain symptoms associated with OA in ACLT-induced rats. The histopathological analysis also showed that oral administration of TCI633 resulted in significant improvement of synovial inflammation, cartilage damage and chondrocytes apoptosis after ACLT surgery. Moreover, oral administration of TCI633 effectively increased the presentation of type II collagen in the cartilage after ACLT. Therefore, it was demonstrated that oral administration of TCI633 could improve disease progression of OA in ACLT-treated rats.

Past studies have demonstrated that the progression of OA is accompanied by pain $(1,3,43)$. Previous in vivo studies have verified that the ACLT model allows clear observation of mechanical allodynia and changes in bipedal weight balance dispersion $(9,10,44)$. Moreover, there will be swelling accompanying the injured knee joint with disease progression after ACLT $(10,44)$. Past studies have verified that hyaluronic acid injections can effectively reduce arthritis pain and has therapeutic effects on OA $(22,45,46)$. In vivo studies have also reported that administering hyaluronic acid can effectively alleviate the disease progression of arthritis caused by ACLT $(5,38)$. A previous study demonstrated that TCI633 can effectively produce hyaluronic acid in the gastrointestinal tract and in blood content (24), and the present study observed that oral administration of TCI633 in ACLT models can alleviate the pain and swelling. Moreover, the TCI633 $\left(5 \times 10^{10}\right.$ or $5 \times 10^{11} \mathrm{CFU} / \mathrm{kg} /$ day) groups and glucosamine treatment groups had similar pain relief effects. Therefore, it was demonstrated that TCI633 can effectively reduce pain and inflammation after ACLT.

The primary causes for OA are long-term mechanical stimulation and the aging process $(2,3)$. Symptoms such as cartilage damage, bone lesions, osteoporosis and synovial tissue inflammation accompany the progression of OA (6). In this study, the histopathological staining showed observations of similar symptoms of human OA after ACLT. The result showed that the synovial and OARSI score were upregulated in the ACLT group. Thus, oral administration of TCI633 and glucosamine could significantly downregulate the index of synovial inflammation and OARSI scores after ACLT. Moreover, administration medium or high doses of TCI633 and glucosamine had superior treatment effects compared with low dose TCI633. Past studies demonstrated that hydrolysis of type II collagen in cartilage would further exacerbate joint destruction of OA $(15,47,48)$. The current study observed that type II collagen expression in cartilage was decreased after ACLT, and administration of TCI633 and glucosamine could increase type II collagen expression in cartilage. Injection of hyaluronic acid has been considered an effective treatment to reduce synovial inflammation and increase collagen II in cartilage after ACLT (49). Previous studies have also reported that the TCI633 administration leads to a delaying bone loss and strengthening bones $(24,27)$. However, it is worth noting that TCI633 also inhibits the gene expression of cathepsin K that plays important role in the hydrolysis of type II collagen $(27,50)$. Therefore, the present study demonstrated that TIC633 can effectively reduce the progression of OA and severity of associated symptoms, and increase type II collagen expression in cartilage after ACLT.

In OA disease progression, there is an association between chondrocyte apoptosis and extracellular matrix loss of cartilage tissue. The main association is that chondrocyte apoptosis changes the synthesis, degeneration and breakage of extracellular matrix in cartilage to form a vicious cycle in OA disease progression $(16,17,51)$. Past studies have demonstrated that increasing type II collagen can affect the survival rate of chondrocytes and injection of hyaluronic acid could improve the rate of chondrocyte apoptosis induced by ACLT $(52,53)$. The present comparisons of the ACLT and Control groups showed a significant increase in the amount of chondrocyte apoptosis. Oral administration of TCI633 and glucosamine effectively reduced the amount of chondrocyte apoptosis. The administration of high doses of TCI633 and glucosamine showed a significant difference with the low dosage TCI633 group. Therefore, the present study demonstrated that oral administration of TCI633 increased type II collagen expression in cartilage and reduced chondrocyte apoptosis to alleviate the effects of OA after ACLT.

Overall, the current study utilized ACLT to induce OA to investigate the therapeutic effects of TCI633 in OA. Nociceptive behaviors and joint swelling were induced in rats by ACLT. The results showed that oral administration of TCI633 effectively alleviated pain and knee joint swelling after ACLT. Simultaneously, medium to high doses of TCI633 showed similar pain relief effects compared with the glucosamine group. Histopathological analysis showed that oral administration of TCI633 can effectively improve the symptoms of OA, including synovial inflammation and cartilage damage with significant therapeutic effects. Moreover, via analyzing type II collagen and chondrocyte apoptosis, oral administration of TCI633 effectively increased type II collagen expression in cartilage and reduced chondrocyte apoptosis to slow the symptoms of OA after ACLT. Thus, the present study demonstrated that oral administration of TCI633 could be useful for suppressing pain and reducing joint swelling through reducing synovial inflammation and cartilage damage in OA. In conclusion, oral administration of TCI633 for long-term use could be used alleviate the symptoms of $\mathrm{OA}$ in humans.

\section{Acknowledgements}

Not applicable.

\section{Funding}

This study was supported by TCI Co., Ltd.

\section{Availability of data and materials}

The datasets used and/or analyzed during the current study are available from the corresponding author on reasonable request. 


\section{Authors' contributions}

YYL, NFC and ZHW conceived and designed the experiments. YYL, PCC, CWF and YWL performed the experiments. YYL and ZHW confirm the authenticity of all the raw data. SNY, YHJ, HMK and YCL analyzed the data. YYL, NFC, YCL and ZHW wrote the paper. The raw data of this study are available from first author and the corresponding author. All authors read and approved the final manuscript.

\section{Ethics approval and consent to participate}

The study was approved by the Institutional Animal Care and Use Committee of National Sun Yat-sen University (approval no. 10417)

\section{Patient consent for publication}

Not applicable.

\section{Competing interests}

The authors declare that they have no competing interests.

\section{References}

1. Litwic A, Edwards MH, Dennison EM and Cooper C: Epidemiology and burden of osteoarthritis. Br Med Bull 105: 185-199, 2013

2. Cross M, Smith E, Hoy D, Nolte S, Ackerman I, Fransen M, Bridgett L, Williams S, Guillemin F, Hill CL, et al: The global burden of hip and knee osteoarthritis: Estimates from the global burden of disease 2010 study. Ann Rheum Dis 73: 1323-1330, 2014

3. Neogi T: The epidemiology and impact of pain in osteoarthritis. Osteoarthritis Cartilage 21: 1145-1153, 2013.

4. Castrogiovanni P, Di Rosa M, Ravalli S, Castorina A Guglielmino C, Imbesi R, Vecchio M, Drago F, Szychlinska MA and Musumeci G: Moderate physical activity as a prevention method for knee osteoarthritis and the tole of dynoviocytes as niological key. Int J Mol Sci 20: 20, 2019.

5. Naito K, Watari T, Furuhata A, Yomogida S, Sakamoto K, Kurosawa H, Kaneko K and Nagaoka I: Evaluation of the effect of glucosamine on an experimental rat osteoarthritis model. Life Sci 86: 538-543, 2010.

6. Cohen-Solal M, Funck-Brentano T and Hay E: Animal models of osteoarthritis for the understanding of the bone contribution. Bonekey Rep 2: 422, 2013.

7. Carmona FD, González-Gay MA and Martín J: Genetic component of giant cell arteritis. Rheumatology (Oxford) 53: 6-18, 2014.

8. Tsai WY, Wu JL, Liu CC, Cherng CH, Tsai RY, Jean YH and Wong CS: Early intraarticular injection of hyaluronic acid attenuates osteoarthritis progression in anterior cruciate ligament-transected rats. Connect Tissue Res 54: 49-54, 2013.

9. Wen ZH, Tang CC, Chang YC, Huang SY, Chen CH, Wu SC, Hsieh SP, Hsieh CS, Wang KY, Lin SY, et al: Intra-articular injection of the selective cyclooxygenase-2 inhibitor meloxicam (Mobic) reduces experimental osteoarthritis and nociception in rats. Osteoarthritis Cartilage 21: 1976-1986, 2013.

10. Wen ZH, Tang CC, Chang YC, Huang SY, Lin YY, Hsieh SP, Lee HP, Lin SC, Chen WF and Jean YH: Calcitonin attenuates cartilage degeneration and nociception in an experimental rat model of osteoarthritis: Role of TGF- $\beta$ in chondrocytes. Sci Rep 6: 28862, 2016.

11. Zhen G, Wen C, Jia X, Li Y, Crane JL, Mears SC, Askin FB, Frassica FJ, Chang W, Yao J, et al: Inhibition of TGF- $\beta$ signaling in mesenchymal stem cells of subchondral bone attenuates osteoarthritis. Nat Med 19: 704-712, 2013.

12. Giunta S, Castorina A, Marzagalli R, Szychlinska MA, Pichler K, Mobasheri A and Musumeci G: Ameliorative effects of PACAP against cartilage degeneration. Morphological, immunohistochemical and biochemical evidence from in vivo and in vitro models of rat osteoarthritis. Int J Mol Sci 16: 5922-5944, 2015.
13. Benito MJ, Veale DJ, FitzGerald O, van den Berg WB and Bresnihan B: Synovial tissue inflammation in early and late osteoarthritis. Ann Rheum Dis 64: 1263-1267, 2005.

14. Rengel Y, Ospelt $\mathrm{C}$ and Gay S: Proteinases in the joint: Clinical relevance of proteinases in joint destruction. Arthritis Res Ther 9: 221, 2007.

15. Eyre D: Collagen of articular cartilage. Arthritis Res 4: 30-35, 2002.

16. Poole AR, Kobayashi M, Yasuda T, Laverty S, Mwale F, Kojima T, Sakai T, Wahl C, El-Maadawy S, Webb G, et al: Type II collagen degradation and its regulation in articular cartilage in osteoarthritis. Ann Rheum Dis 61 (Suppl 2): ii78-ii81, 2002.

17. Hwang HS and Kim HA: Chondrocyte apoptosis in the pathogenesis of osteoarthritis. Int J Mol Sci 16: 26035-26054, 2015.

18. Wu L and Liu Z: The molecular mechanisms of preventing apoptosis of cartilage chondrocyte to target osteoarthritis. Future Med Chem 9: 537-540, 2017.

19. Musumeci G, Castrogiovanni P, Loreto C, Castorina S, Pichler K and Weinberg AM: Post-traumatic caspase-3 expression in the adjacent areas of growth plate injury site: A morphological study. Int J Mol Sci 14: 15767-15784, 2013.

20. Musumeci G, Loreto C, Carnazza ML and Martinez G: Characterization of apoptosis in articular cartilage derived from the knee joints of patients with osteoarthritis. Knee Surg Sports Traumatol Arthrosc 19: 307-313, 2011

21. Lespasio MJ, Piuzzi NS, Husni ME, Muschler GF, Guarino A and Mont MA: Knee Osteoarthritis: A Primer. Perm J 21: 16-183, 2017.

22. Sinusas K: Osteoarthritis: Diagnosis and treatment. Am Fam Physician 85: 49-56, 2012

23. Ma VY, Chan L and Carruthers KJ: Incidence, prevalence, costs, and impact on disability of common conditions requiring rehabilitation in the United States: Stroke, spinal cord injury, traumatic brain injury, multiple sclerosis, osteoarthritis, rheumatoid arthritis, limb loss, and back pain. Arch Phys Med Rehabil 95: 986-995.e1, 2014

24. Lin YH, Su HL and Yu CH: Method of enhancing hyaluronic acid secretion using probiotic strain. US Patent US9289455B2. Filed March 26, 2015; issued October 15, 2015.

25. Ogita T, Nakashima M, Morita H, Saito Y, Suzuki T and Tanabe S: Streptococcus thermophilus ST28 ameliorates colitis in mice partially by suppression of inflammatory Th17 cells. J Biomed Biotechnol 2011: 378417, 2011.

26. Ai C, Zhang Q, Ren C, Wang G, Liu X, Tian F, Zhao J, Zhang H, Chen YQ and Chen W: Genetically engineered Lactococcus lactis protect against house dust mite allergy in a BALB/c mouse model. PLoS One 9: e109461, 2014.

27. Lin YH, Chen IH, Shih HH and Lee CY: Method for preventing and/or treating osteoporosis by using Streptococcus thermophilus TC1633 strain and its metabolites. US Patent US10149871B2. Filed June 21, 2017; issued December 21, 2017.

28. Balazs EA and Denlinger JL: Viscosupplementation: A new concept in the treatment of osteoarthritis. J Rheumatol Suppl 39: 3-9, 1993

29. Shimizu C, Yoshioka M, Coutts RD, Harwood FL, Kubo T, Hirasawa Y and Amiel D: Long-term effects of hyaluronan on experimental osteoarthritis in the rabbit knee. Osteoarthritis Cartilage 6: 1-9, 1998

30. Abatangelo G, Botti P, Del Bue M, Gei G, Samson JC, Cortivo R, De Galateo A and Martelli M: Intraarticular sodium hyaluronate injections in the Pond-Nuki experimental model of osteoarthritis in dogs. I. Biochemical results. Clin Orthop Relat Res 241: 278-285, 1989.

31. Yoshimi T, Kikuchi T, Obara T, Yamaguchi T, Sakakibara Y, Itoh $\mathrm{H}$, Iwata $\mathrm{H}$ and Miura $\mathrm{T}$ : Effects of high-molecular-weight sodium hyaluronate on experimental osteoarthrosis induced by the resection of rabbit anterior cruciate ligament. Clin Orthop Relat Res 298: 296-304, 1994.

32. Altman RD and Moskowitz R; Hyalgan Study Group: Intraarticular sodium hyaluronate (Hyalgan) in the treatment of patients with osteoarthritis of the knee: A randomized clinical trial. J Rheumatol 25: 2203-2212, 1998.

33. Amiel D, Toyoguchi T, Kobayashi K, Bowden K, Amiel ME and Healey RM: Long-term effect of sodium hyaluronate (Hyalgan) on osteoarthritis progression in a rabbit model. Osteoarthritis Cartilage 11: 636-643, 2003.

34. Tamoto K, Tada M, Shimada S, Nochi H and Mori Y: Effects of high-molecular-weight hyaluronates on the functions of guinea pig polymorphonuclear leukocytes. Semin Arthritis Rheum 22 (Suppl 1): 4-8, 1993. 
35. Sheehan KM, DeLott LB, Day SM and DeHeer DH: Hyalgan has a dose-dependent differential effect on macrophage proliferation and cell death. J Orthop Res 21: 744-751, 2003.

36. Ghosh P, Read R, Numata Y, Smith S, Armstrong S and Wilson D: The effects of intraarticular administration of hyaluronan in a model of early osteoarthritis in sheep. II. Cartilage composition and proteoglycan metabolism. Semin Arthritis Rheum 22 (Suppl 1): 31-42, 1993.

37. Yang PY, Tang CC, Chang YC, Huang SY, Hsieh SP, Fan SS, Lee HP, Lin SC, Chen WF, Wen ZH, et al: Effects of tibolone on osteoarthritis in ovariectomized rats: Association with nociceptive pain behaviour. Eur J Pain 18: 680-690, 2014.

38. Wen ZH, Tang CC, Chang YC, Huang SY, Hsieh SP, Lee CH, Huang GS, Ng HF, Neoh CA, Hsieh CS, et al: Glucosamine sulfate reduces experimental osteoarthritis and nociception in rats: Association with changes of mitogen-activated protein kinase in chondrocytes. Osteoarthritis Cartilage 18: 1192-1202, 2010.

39. Fernihough J, Gentry C, Malcangio M, Fox A, Rediske J, Pellas T, Kidd B, Bevan S and Winter J: Pain related behaviour in two models of osteoarthritis in the rat knee. Pain 112: 83-93, 2004.

40. Chaplan SR, Bach FW, Pogrel JW, Chung JM and Yaksh TL: Quantitative assessment of tactile allodynia in the rat paw. J Neurosci Methods 53: 55-63, 1994.

41. Pritzker KP, Gay S, Jimenez SA, Ostergaard K, Pelletier JP, Revell PA, Salter D and van den Berg WB: Osteoarthritis cartilage histopathology: Grading and staging. Osteoarthritis Cartilage 14: 13-29, 2006.

42. Lee $\mathrm{CH}$, Wen $\mathrm{ZH}$, Chang YC, Huang SY, Tang CC, Chen WF, Hsieh SP, Hsieh CS and Jean YH: Intra-articular magnesium sulfate $\left(\mathrm{MgSO}_{4}\right)$ reduces experimental osteoarthritis and nociception: Association with attenuation of N-methyl-D-aspartate (NMDA) receptor subunit 1 phosphorylation and apoptosis in rat chondrocytes. Osteoarthritis Cartilage 17: 1485-1493, 2009.

43. Bartley EJ, Palit S and Staud R: Predictors of osteoarthritis pain: The importance of resilience. Curr Rheumatol Rep 19: 57, 2017.

44. Kao JH, Lin SH, Lai CF, Lin YC, Kong ZL and Wong CS: Shea nut oil triterpene concentrate attenuates knee osteoarthritis development in rats: Evidence from knee joint histology. PLoS One 11: e0162022, 2016.
45. Monticone M, Frizziero A, Rovere G, Vittadini F, Uliano D, LA Bruna S, Gatto R, Nava C, Leggero V and Masiero S: Hyaluronic acid intra-articular injection and exercise therapy: Effects on pain and disability in subjects affected by lower limb joints osteoarthritis. A systematic review by the Italian Society of Physical and Rehabilitation Medicine (SIMFER). Eur J Phys Rehabil Med 52: 389-399, 2016.

46. Hashizume M, Koike N, Yoshida H, Suzuki M and Mihara M: High molecular weight hyaluronic acid relieved joint pain and prevented the progression of cartilage degeneration in a rabbit osteoarthritis model after onset of arthritis. Mod Rheumatol 20: 432-438, 2010.

47. Stoop R, Buma P, van der Kraan PM, Hollander AP, Billinghurst RC, Meijers TH, Poole AR and van den Berg WB: Type II collagen degradation in articular cartilage fibrillation after anterior cruciate ligament transection in rats. Osteoarthritis Cartilage 9: 08-315, 2001.

48. Bakilan F, Armagan O, Ozgen M, Tascioglu F, Bolluk O and Alatas O: Effects of native type II collagen treatment on knee osteoarthritis: A randomized controlled trial. Eurasian J Med 48: 95-101, 2016.

49. Zhang Z, Wei X, Gao J, Zhao Y, Zhao Y, Guo L, Chen C, Duan Z, Li P and Wei L: Intra-articular injection of cross-linked Hyaluronic acid-dexamethasone hydrogel attenuates osteoarthritis: An experimental study in a rat model of osteoarthritis. Int J Mol Sci 17: 411, 2016.

50. Dejica VM, Mort JS, Laverty S, Percival MD, Antoniou J, Zukor DJ and Poole AR: Cleavage of type II collagen by cathepsin K in human osteoarthritic cartilage. Am J Pathol 173: 161-169, 2008.

51. Aigner T and Kim HA: Apoptosis and cellular vitality: Issues in osteoarthritic cartilage degeneration. Arthritis Rheum 46: 1986-1996, 2002.

52. Lotz M, Hashimoto S and Kühn K: Mechanisms of chondrocyte apoptosis. Osteoarthritis Cartilage 7: 389-391, 1999.

53. Barreto RB, Sadigursky D, de Rezende MU and Hernandez AJ: Effect of hyaluronic acid on chondrocyte apoptosis. Acta Ortop Bras 23: 90-93, 2015. 